\title{
LES FEMMES DANS LE PARTI DES TRAVAILLEURS (PT) À SÃO PAULO (BRÉSIL) Vers l'émergence d'une oligarchie féminine?
}

L'organisation des femmes au sein du PT, à São Paulo, suit une évolution comparable à celle que connaît le parti dans son ensemble. On assiste en effet à une professionnalisation et une bureaucratisation du PT contribuant à la création d'une oligarchie partisane masculine. À l'échelle interne, l'institutionnalisation des problématiques de genre, destinée à redéfinir les rapports de pouvoir entre les sexes, aboutit à la naissance d'une oligarchie féminine restreinte, dont le manque de légitimité symbolise les difficultés que rencontrent les femmes politiques lorsqu'elles prétendent représenter l'universel.

\section{As mulheres no Partido dos Trabalhadores (PT) em São Paulo (Brasil): Rumo à emergência de uma oligarquia feminina?}

A organização das mulheres no seio do PT, em São Paulo, segue uma evolução comparável à que conhece o partido no seu conjunto. Assistimos com efeito a uma profissionalização e a uma burocratização do PT, contribuindo para a criação de uma oligarquia partidária masculina. No seio do partido, a institucionalização das problemáticas de género, cujo objectivo é a redefinição das relações de poder entre os sexos, resulta na criação de uma oligarquia feminima restrita, cuja falta de legitimidade simboliza as dificuldades que as mulheres políticas enfrentam quando pretendem representar o universal.

\section{Women in the Workers' Party (PT) in Sao Paulo (Brazil): \\ Towards an Emerging Female Oligarchy?}

The organization of women within the Worker's Party shows an evolution which is analogous to the changes that have occured in the party as a whole. Its bureaucratization and professionalisation have indeed led to the creation of a masculine oligarchy. The institutionalization of gender claims, aimed at reshaping the sexual relations of power at an internal level, have given rise to a restricted female oligarchy whose lack of legitimacy symbolizes the difficulties encountered by political women in their claim to a universal representation.

$\mathrm{L}$ e développement des études traitant du militantisme féminin contribue à mettre en lumière les rapports de pouvoir entre les sexes au sein des mouvements sociaux et organisations politiques. Rompant ainsi avec une image androcentrée du militantisme, elles tendent, pour certaines d'entre elles, à se concentrer sur l'hétérogénéité des pratiques, alimentant l'idée selon laquelle «les femmes font de la politique autrement». Mon étude sur le Secrétariat national des femmes (SNF) du Parti des Travailleurs (PT), à São Paulo était ainsi motivée par la volonté d'analyser les variations de pratiques militantes, selon le genre, au sein d'un parti 
politique ${ }^{1}$. Aussi ai-je été surprise de constater qu'au lieu de distinctions, je rencontrai, tout d'abord, une déconcertante similarité entre l'évolution de la structuration du PT et celle de l'organisation des femmes dans ce dernier.

Deux niveaux d'analyse ont été privilégiés pour cette étude. Tout d'abord, j’ai appréhendé le PT comme une entité collective. Les analyses réalisées dans les années 1980 insistent sur la singularité de ce parti tant sur la scène politique brésilienne, qu'en rapport à la littérature sur les partis politiques. Ce parti «pas comme les autres » selon Orlando Fals Borda ${ }^{2}$, permettait de questionner la distinction traditionnellement établie entre l'arène sociale institutionnalisée et l'espace des conflits sociaux ${ }^{3}$, la distinction entre ces deux champs étant particulièrement ténue dans le cas du PT. Étudier cette même organisation en 2004 ne conduit bien évidemment pas aux mêmes conclusions : en investissant le champ institutionnel et en privilégiant progressivement ce dernier, le PT s'apparente de plus en plus à un parti politique classique. Il confirme l'existence d'une «loi d'airain de l'oligarchie $»^{4}$, liée à un processus de professionnalisation et de bureaucratisation partisane, évolution allant de pair avec une loi d'airain de la patriarchie.

Ensuite, le PT est analysé comme une relation sociale, une «sociation » selon la terminologie wéberienne, afin de considérer les dynamiques de pouvoir à l'échelle interne. En tant qu'«association complexe de groupements en interaction et en concurrence pour l'imposition de modèles légitimes des pratiques et des comportements $»^{5}$, le PT connaît un ensemble de processus différenciés selon les groupes, tendances et courants. En effet, le phénomène d'institutionnalisation et de bureaucratisation ne revêt pas la même ampleur et ne suit pas la même temporalité selon les groupements intra partisans. Une étude du PT, depuis sa formation, aboutit à la mise en lumière de «vagues d'institutionnalisation ».

L'introduction de la notion de genre dans la littérature traitant des partis politiques s'est révélée particulièrement heuristique. Joan Scott est, selon moi, celle qui définit le mieux la portée de ce concept. Selon cette auteure, le genre est un « élément constitutif des rapports sociaux fondés sur des différences perçues entre les sexes et [...] une première façon de signifier les rapports de pouvoir $»^{6}$.

1 Cet article est une partie de mon mémoire de DEA, réalisé sous la direction de M. Daniel van Eeuwen, pour lequel j’ai effectué une étude de terrain durant cinq semaines à São Paulo en avril-mai 2004.

2 O. Fals Borda, «Mouvements sociaux et pouvoir politique: développement en Amérique latine », in A. Alvarez Bejar et al. (eds), Amérique latine, démocratie et exclusion, Paris, l'Harmattan, 1994 : 210.

${ }^{3}$ Erik Neveu met en garde contre les dangers d'une dichotomie simplificatrice entre arène sociale institutionnalisée et arène des conflits sociaux. Cette distinction reste, toutefois, intéressante si l'on adopte une conception relationnelle du pouvoir. E. Neveu, Sociologie des mouvements sociaux, Paris, La Découverte, 2002 : 17-20 («Repères »).

4 R. Michels, Les Partis politiques, essais sur les tendances oligarchiques des démocraties, Paris, Champs Flammarion, 1984, 341 p. [Leipzig, 1911]

${ }^{5}$ J. Lagroye, Sociologie politique, Paris, Presses de Sciences Po et Dalloz, 1997 : 273-274. (3e éd.)

${ }^{6}$ J. Scotт, «Genre : une catégorie utile d'analyse historique », Les cahiers du GRIF, "Le Genre de l'histoire", 37-38, 1988 : 125-153. 
L'objectif de cet article consiste bien plus à proposer des pistes de recherche qu'à formuler de fermes conclusions ${ }^{7}$. Mon hypothèse est la suivante : en réaction à l'« oligarchisation » de l'organisation partisane, réalisée au profit d'une élite masculine, le setorial ${ }^{8}$ aux femmes du PT se mobilise pour une institutionnalisation des problématiques de genre et une remise en question des rapports de pouvoir partisan. Ce processus mène à la naissance d'une oligarchie féminine, dont la légitimité fait l'objet d'une forte remise en question.

Similarité des processus, disais-je en début d'introduction, ou peut être que le terme le plus approprié serait analogie ${ }^{9}$, puisqu'il s'agit de la reproduction genrée d'un «mécanisme » observable au sein d'une variété d'organisations politiques. En tant que «même dans l'autre ou autre dans le même $»^{10}$, cette analogie permet, en outre, de mettre en lumière les distinctions entre élite féminine et masculine.

Une dernière précision méthodologique s'impose quand au choix de São Paulo comme terrain d'étude. En raison des origines du PT, de la présence de la direction nationale et de l'ampleur du mouvement féministe dans cette même ville lors de la création du parti, São Paulo semblait être le terrain privilégié. Les hypothèses présentées dans cet article sont difficilement généralisables au-delà du cas étudié du fait de la diversité des dynamiques politiques régionales et de l'hétérogénéité du parti. L'utilisation du terme PT, dans cet article répond à un souci de simplification mais n'élude pas le caractère localisé de cette étude.

Nous considérerons, dans un premier temps l'évolution de la structuration du PT : l'investissement progressif de l'arène classique institutionnalisée s'accompagne d'une bureaucratisation du parti au profit d'une oligarchie masculine. Puis, la dynamique interne du PT sera l'objet de notre attention: l'institutionnalisation des problématiques de genre suscite une "oligarchisation» féminine, cependant peu légitime.

\section{De la mobilisation multisectorielle à l'institutionnalité politique : panorama à deux voix de l'évolution de la structuration du PT}

Les mutations de la structuration partisane du PT, en tant qu'organisation ou entreprise collective, traduisent l'évolution des priorités du parti. L'arène institutionnelle est aujourd'hui privilégiée, au détriment de l'arène des conflits sociaux.

La création du PT, le 14 octobre 1979, polarise une grande partie des forces d'opposition à la dictature militaire durant laquelle le bipartisme artificiellement créé ne traduisait que très peu les clivages socio-économiques de la société brésilienne $^{11}$. Les partis politiques étant traditionnellement créés «par le haut», le PT se distingue par son assise sociale. La naissance de ce dernier repose sur le

${ }^{7}$ Cet article représente en effet un premier essai de conceptualisation, susceptible d'évoluer à l'issue de nouvelles recherches.

${ }^{8}$ Les setoriais ou secteurs désignent les groupes thématiques constitués au sein du PT.

9 Philippe Corcuff propose de s'émanciper de l'opposition figée « Même/Autre » par laquelle on envisage les phénomènes sous l'angle de l'identité et de la différence, à travers la figure de l'Analogue. P. Corcuff, Philosophie politique, Paris, Nathan Université, 2000, 128 p. (Col. « 128 »).

${ }_{10}$ P. Corcuff, Philosophie politique, op. cit. : 15.

11 Sous la dictature militaire, seuls deux partis étaient autorisés : l'Arena (Alliance rénovatrice nationale), parti du gouvernement et le MDB, parti d'opposition. Il devient PMDB en 1979. 
développement du mouvement ouvrier autour du nouveau syndicalisme ${ }^{12}$ ainsi que sur le soutien de plusieurs composantes sociales ${ }^{13}$ : les parlementaires de la Gauche du MDB (Movimento Democrático Brasileiro) marginalisés au sein de leur parti, plusieurs intellectuels, des organisations d'extrême gauche ainsi qu'un nombre significatif de mouvements populaires urbains, en grande partie créés sous la tutelle des secteurs progressistes de l'Église catholique. Parmi ce dernier type d'acteurs, les mouvements de femmes furent particulièrement actifs. Selon Sonia Alvarez, les organisations féminines ayant été relativement épargnées par le pouvoir militaire, en raison d'une perception stéréotypée des femmes reposant sur leur caractère prétendument apolitique, elles purent se développer et s'implanter au sein de l'arène des conflits sociaux ${ }^{14}$. En 1979, alors que le PT est en formation, le mouvement féministe connaît son apogée, en particulier à São Paulo, et tente de réaliser une unité avec les divers mouvements de femmes, c'est-à-dire les mouvements populaires et de lutte pour la démocratisation ${ }^{15}$. À la différence des partis socialistes et communistes d'Europe occidentale, le PT est créé alors que les femmes ont massivement investi l'espace public.

L'importance des mobilisations féminines dans la ville de São Paulo et dans ses alentours (Santos et Agudo notamment) pour la consolidation locale du $\mathrm{PT}^{16}$ est une variable à prendre en considération si l'on veut comprendre l'introduction des thématiques de genre dans le projet du parti et l'organisation des femmes au sein de ce dernier.

Le projet du PT est fortement influencé par l'expérience du nouveau syndicalisme et sa volonté de redéfinir des liens entre la société et l'État avec comme horizon la socialisation de la politique. Une structuration complexe est envisagée reposant sur les principes d'organisation et de participation des bases et à l'origine d'une décentralisation de la prise de décision. À cette fin, des Nucleos (Noyaux) de deux sortes - mixtes et strictement féminins - sont envisagés. Par ailleurs, les féministes du parti, formant d'abord un collectif à São Paulo, repoussent l'idée de créer une structure propre au sein du PT pour ne pas reproduire la dichotomie classique entre thématiques "particulières » de genre et thématiques «générales », les premières devant être constamment intégrées aux secondes :

12 Le syndicalisme classique brésilien, né au début du siècle est rapidement encadré et contrôlé par le gouvernement Vargas. Le «nouveau syndicalisme » se distingue de ce dernier par les secteurs qu'il mobilise (grandes entreprises modernes de métallurgie, d'automobile) ainsi que par une attitude d'autonomie à l'égard des forces politiques.

13 R. Meneguello, PT: A formação de um partido, 1979-1982, São Paulo, editora Paz e Terra, $1989: 45$.

14 S. Alvarez, Engendering Democracy in Brazil. Women's movement in transition, Princeton N.J., Princeton University Press, 1990 : 60.

${ }^{15}$ Cette classification des mouvements de femmes, en Amérique latine, est proposée par Bérengère Marques-Pereira et Florence Raes. B. Marques-Pereira \& F. Raes, «Trois décennies de mobilisations féminines en Amérique latine: une évaluation des avancées, limites et des futurs enjeux de l'action collective des femmes », Cahiers des Amériques latines, Paris, Institut des hautes études de l'Amérique latine, 39, 2002/1: 17-36.

${ }^{16}$ F. Mac Caulay, «The purple in the Rainbow: Gender Politics in the PT », in G. Baiocchi (ed.), Radicals in Power. The Worker's Party and Experiments in Urban Democracy in Brasil, Londres, Zed Books, 2003 : 176-201. 
«Nous ressentons la nécessité de discuter de la participation des femmes dans le PT: c'est-à-dire que nous sommes peut-être le seul parti sans département féminin. [...] En premier lieu, la femme doit travailler au coude à coude avec les hommes du parti [...] En second lieu, il y a un mouvement qui grandit, il serait absurde d'avoir un département féminin ou une autre instance qui engloutisse le mouvement au lieu de la soutenir $»^{17}$.

Bien qu'une autonomie statutaire soit laissée aux syndicats et aux mouvements sociaux, et ce afin de rompre avec l'encadrement historique et l'utilisation stratégique de ces derniers, le PT entretient des liens très étroits avec plusieurs organisations politiques si bien que dans un premier temps, de sa création à 1985, il se définit comme un instrument de participation aux mains des travailleurs pour l'accès aux institutions politiques. Le revers électoral des premières élections libres, à l'échelon municipal en 1982, ainsi qu'une division au sein du mouvement syndical à l'origine de la Central Unica de los Trabalhadores (CUT), contribue en outre à un mouvement de «retour aux bases» durant lequel l'activité syndicale et sociale constitue l'essentiel du travail des militants. Les mouvements sociaux représentent une source de légitimité partisane et renforcent la crédibilité d'un parti encore en voie de consolidation. Des tensions entre la direction partisane et les élus parlementaires apparaissent néanmoins à cette époque, lesquelles s'accentueront par la suite avec la croissance du parti et les victoires électorales ${ }^{18}$. À São Paulo, les expériences de gestion municipale coïncident avec une institutionnalisation du parti et un investissement progressif de l'arène politique. Dans le même temps, la structure partisane s'«oligarchise » et se professionnalise, confirmant l'existence d'une loi d'airain de la patriarchie.

\section{Une « loi d'airain de l'oligarchie » et de la patriarchie}

L'implantation du PT sur la scène politique brésilienne s'accompagne d'une évolution du profil du parti, corroborant l'existence d'une loi d'airain de l'oligarchie mise en lumière dès 1911 par Roberto Michels ${ }^{19}$. Selon cet auteur, le principe de délégation, inévitable et nécessaire dans une organisation, aboutit à la création d'une élite dirigeante, dont les intérêts se distinguent progressivement de la base militante. À mesure que le parti s'accroît, la bureaucratie se consolide et se professionnalise au bénéfice des militants dotés d'une formation intellectuelle qu'ils peuvent mettre à profit au sein de l'organisation. On observe bien une évolution de la composition des instances de direction en faveur des classes moyennes ${ }^{20}$,

${ }^{17}$ Comissão de Mulheres, A participação do PT no III congresso da mulher Paulista, 1981, multigr.

${ }^{18}$ M. Keck, The Worker's Party and Democratisation in Brazil, New Haven, Yale University Press, 1992: 180-190.

${ }^{19}$ R. Michels, Les Partis Politiques,..., op. cit.

${ }^{20} \mathrm{La}$ progression des classes moyennes, en particulier des enseignants et des banquiers au sein de la GUT, joue un rôle non négligeable dans cette évolution. Cf. D. SAmuels, «From Socialism to Social Democracy. Party Organisation and the Transformation of the Worker's Party in Brazil », Comparative Political Studies (Thousand Oaks, Sage Publications), XXXVII (9), November 2004: 999-1024. 
ainsi qu'une professionnalisation d'un noyau dur de dirigeants ${ }^{21}$ entretenant d'étroits liens avec les organisations syndicales. Cette évolution ne doit pas être surestimée : soumis à un certain contrôle de la part des «bases", les dirigeants nationaux connaissent une réelle autonomisation à partir de $1995^{22}$. La réforme des statuts de $2000^{23}$ accroît le rôle des militants pour la sélection des dirigeants au détriment des tendances internes. Il est encore trop tôt pour analyser les conséquences d'une telle réforme. On peut néanmoins se demander si, en redéfinissant les modalités de la compétition interne, jusqu'alors fortement institutionnalisée au profit d'une concurrence entre tendances et, partant de cela, favorisant une certaine rotation des élites, l'«oligarchisation » ne va pas s'en trouver renforcée.

Cette tendance confirme également l'existence d'une «loi d'airain de la patriarchie » : avec l'« oligarchisation » du parti, les possibilités d'ascension des femmes se réduisent. Au début des années 1980, la structure partisane étant encore lâche, le PT fait appel aux initiatives individuelles et collectives offrant davantage d'opportunités aux candidatures féminines. Ainsi en 1982, le PT est le deuxième parti en terme de féminisation des listes et le premier en matière de candidates issues des mouvements de femmes. Dans la ville de São Paulo, trois candidates sont élues alors que le nombre de vereadoras petistas ${ }^{24}$ s'élève à cinq: Irede Cardoso, dont le programme était centré sur les problématiques féministes, Teresa Lajolo et Luiza Erundina, toutes deux militantes de mouvements populaires de femmes. Aujourd'hui, le pourcentage d'élues petistas, à l'échelle nationale, avoisine les $15 \%$.

Les facteurs d'explication de la reproduction d'une élite partisane - reposant sur l'accumulation de privilèges statutaires, d'information et de notoriété selon Philippe Braud ${ }^{25}$ - nous éclairent, imparfaitement néanmoins, sur l'existence d'un «plafond de verre » au sein des organisations partisanes.

La commission nationale provisoire créée en 1979, était composée d'acteurs en mesure de mobiliser des ressources variées. Certains dirigeants bénéficiaient d'une forte légitimité découlant de leur rôle durant les grèves des métallurgistes de 19781979 (Lula) ainsi que de leur expérience syndicale (Lula, Olivier Dutra, Jacó Bittar étaient présidents de syndicats), expérience leur conférant des compétences précieuses reconverties en capital politique au sein du PT. L'exercice d'une responsabilité parlementaire (Edson Khair, ancien député du MDB) ou la détention de titres universitaires (Firmo Trinidade, économiste) constituent également une source de légitimité. Dans cette perspective, seules quelques femmes, ayant des ressources de pouvoir comparables, réussirent à intégrer le noyau dirigeant lors de la phase de constitution du parti : Irma Passoni, ancienne députée du MDB de l'État de São Paulo, Clara Charf, militante communiste et féministe exilée durant la dictature et veuve du révolutionnaire Marighella, et l'avocate Helena Grecco.

21 R. Meneguello, "The Dilemmas and Limit of Transformation : a Commentary », in G. Baiocchi (ed.), Radicals in Power. ., op. cit. : 217.

${ }^{22}$ D. Samuels, « From Socialism ... », op. cit. : 1019.

${ }^{23}$ Celle-ci modifie le mode d'élection de la direction nationale au profit d'une élection directe par les adhérents, d'un allongement des mandat (trois ans au lieu de deux) et d'une limitation à trois mandats.

${ }^{24}$ Ce titre est l'équivalent de conseillère municipale du PT.

25 P. Braud, Sociologie politique, Paris, LGDJ, 2003 : 449-442. (6 éd.) 
Toutefois, ces cas représentent bien plus l'exception que la règle : l'investissement au sein de mouvements sociaux est inégalement converti en ressources politiques. Arguant d'un sentiment d'incompétence, plusieurs des militantes rencontrées, ayant eu une expérience significative de militantisme au préalable, au sein de mouvements sociaux urbains notamment, ont préféré acquérir de solides connaissances politiques avant de s'engager dans la compétition politique. «Éternelle administration de la preuve $»^{26}$, conduisant les femmes à s'astreindre à un sur-travail avant d'affronter leurs compères masculins ou réel manque de formation politique, il est souvent difficile de déterminer quelle variable est la plus significative. L'entremêlement entre caractéristiques de genre et insuffisantes ressources politiques est fort ${ }^{27}$ : l'engagement dans le parti avec des ressources peu valorisées et converties en capital politique explique que les femmes du collectif agissent comme si elles étaient des outsiders souhaitant changer les règles du jeu et se repliant sur les problématiques de genre, contribuant, en outre, à l'institutionnalisation de ces dernières.

\section{L'institutionnalisation des problématiques de genre au sein du PT}

Si le PT intègre, à l'origine, dans son discours et son programme le principe de l'enchevêtrement des problématiques de genre et de classe, l'engagement du parti en la matière décroît à mesure que ce dernier s'implante sur la scène politique brésilienne. On assiste en effet, à une baisse du nombre de femmes au sein des instances de direction, en particulier à la fin des années 1990. Les revendications des mouvements féminins connaissent également un écho moins important dans le programme du parti ${ }^{28}$. Afin de contrebalancer cette évolution, les femmes petistas s'organisent autour de deux revendications: l'adoption de quotas de $30 \%$ de représentation féminine pour les instances de direction; la création d'un secrétariat des femmes.

L'adoption de quotas en 1991 découle, en premier lieu, d'une forte mobilisation des féministes petistas et fut, progressivement soutenue par la grande majorité des militantes du parti. Ces quotas sont approuvés alors que le taux de représentation féminine interne est de $6 \%$ et sont strictement respectés depuis lors.

Le processus d'institutionnalisation de l'organisation des femmes suit, en second lieu, une évolution progressive: il existait tout d'abord un collectif de femme à São Paulo abrité par le secrétariat des mouvements populaires. À partir des années 1990, apparaît un sous-secrétariat des Femmes, devenu SNF en 1996. Celui-ci est créé alors que la structuration partisane se complexifie et reconnaît l'existence de divers setoriais. En 1994 sont formés les secrétariats de combat contre le racisme et le secrétariat pour la protection de l'environnement. Cette «vague d'institutionnalisation » mérite notre attention. Elle met en lumière l'existence de processus

${ }^{26}$ M. Sineau, Des femmes en politique, Paris, Economica, 1988 : 109 (« La Vie Politique »).

${ }^{27}$ Cette corrélation est mise en lumière par Catherine Achin et Marion Paoletti pour le cas des élues municipales à la suite de l'adoption de la loi sur la parité. Cf. C. Achin \& M. Paoletti, «Le salto du stigmate », Politix (Paris), l'Harmattan, 60, 2001 : 31-45.

${ }^{28}$ La question de l'avortement est à ce titre significative : le PT défendait en 1982 la légalisation de l'avortement, position qui a, ensuite, fait l'objet d'engagements bien plus nuancés voire contradictoires selon les déclarations. 
différenciés au sein du parti. Le secteur femmes est caractérisé, durant ses premières années d'existence, par une évolution distincte des instances les plus influentes du parti. Les phénomènes de bureaucratisation et de professionnalisation semblaient bien moins caractériser cette structure, pour le moins jusqu'aux victoires électorales locales et nationales du PT au début des années 2000.

Le SNF décide, d'abord, de définir ses propres règles de fonctionnement, dérogeant en partie aux statuts du parti : l'élection d'une représentante siégeant à la Commission exécutive nationale était réalisée par les membres du secteur femmes, afin de préserver le principe de non « oligarchisation» de la structure ${ }^{29}$.

«En 1995, il [collectif de femmes] se détache des mouvements populaires et se constitue en secrétariat. Au début il y avait une philosophie quasi innocente [. . . Personne ne devait prendre la direction du secrétariat, toutes les décisions devaient être prises au consensus, il devait toujours exister une horizontalité du pouvoir...» (Membre de la Coordenadoria da mulher ${ }^{30}$ de São Paulo).

De plus, les oppositions entre tendances n'étaient pas inexistantes au sein du SNF mais ne constituaient pas une source de division ou de positionnement distinct. Ce fonctionnement collégial était assorti d'une priorité accordée au renforcement des mouvements de femmes, alors que, dans le même temps, la Direction nationale $(\mathrm{DN})$ tendait à privilégier l'investissement de l'espace institutionnel ${ }^{31}$.

«Dans le PT, on considère que les collectifs de femmes sont encore des domaines très militants, très volontaires, qui mobilisent beaucoup de personnes, car le reste est très lié aux cabinets, aux députés, aux conseillers municipaux...» (Secrétaire aux femmes de l'État de São Paulo).

Les mouvements sociaux de femmes représentent une ressource pour le SNF. En effet, la création de structures proprement féminines augmente les capacités d'action des militantes en favorisant l'émergence d'une identité commune, en fournissant un accès aux instances de pouvoir ${ }^{32}$ et des fonds propres pour la réalisation de projet. Néanmoins, le SNF n'exerce qu'une influence secondaire au sein de la dynamique partisane. Du fait de cette faiblesse, l'appui des mouvements sociaux permet de pallier l'illégitimité interne. Ce soutien est d'autant plus important qu'il s'inscrit dans la logique du projet du parti : l'utilisation du discours partisan sur lui-même - une organisation créée par et pour les travailleurs -, permet aux féministes petistas de légitimer leurs revendications.

L'organisation des secrétariats ainsi que les relations entretenues avec les mouvements sociaux corroborent-elles l'idée qu'il existe des pratiques politiques «gen-

29 Selon l'article $112 \oint 1$ des statuts du parti, il revient à la direction nationale d'élire les représentants des divers secrétariats, notamment des secteurs du parti, « en accord avec ses membres ». Cette dernière précision justifie, pour le secteur femmes, l'élection d'une représentante par ses propres membres.

30 Coordination de la femme.

31 La «distanciation de la direction à l'égard de ses bases » est reconnue dès 1987, dans la résolution «La construction du PT», Partido dos Trabalhadores, resoluções e congressos. 1979-1998, São Paulo, Fundação Perseu Abramo: 349.

32 Une déléguée siège au sein de la Commission exécutive nationale avec un pouvoir de consultation. En 2001, elle acquiert un droit de vote au sein de cette instance. 
rées » ou découlent-elles de l'investissement d'arènes "socialement dominées » au sein desquelles les rétributions sont faibles? Existent-ils des différences de pratiques selon le genre lorsque les arènes investies par les femmes sont l'objet d'enjeux de pouvoir? Les victoires électorales du PT à l'échelle municipale en 2000 et nationale en 2002 et l'intégration, certes limitée, des problématiques de genre sur l'agenda politique des nouveaux gouvernements, coïncident avec la naissance de tensions au sein des secrétariats et une certaine «oligarchisation » féminine.

\section{Quelle légitimité pour une oligarchie féminine?}

Faire l'hypothèse de la naissance d'une oligarchie féminine suppose quelques précautions: les femmes ne détiennent pas l'autorité partisane et restent largement sous-représentées au sein du PT. Néanmoins, ce que je souhaite mettre en lumière est l'existence d'une «loi d'airain de l'oligarchie » distincte selon le genre, l'élite féminine naissante étant soumise à une double remise en question de sa légitimité.

Deux facteurs ont contribué à la naissance d'une oligarchie féminine au sein du parti : l'adoption de quotas et la mise en place de structures chargées d'élaborer des politiques publiques en faveur des femmes.

Tout d'abord, l'adoption d'un quota de $30 \%$ de représentation féminine au sein des directions partisanes mène à un renouvellement des cadres partisans, au profit de militantes généralement dotées de capitaux culturel et politique élevés. Il semblerait qu'à São Paulo, les élites féminines soient relativement diplômées et travaillent majoritairement dans l'éducation ou les métiers du «social » ${ }^{33}$. Bien que les femmes profitent inégalement de leur expérience au sein des instances de direction, cette mesure permet à quelques-unes de s'implanter durablement au sein de ces arènes: les cas d'Arlete Avelar Sampaio, Clara Ant et Iriny Lopes ${ }^{34}$, entre autres, sont révélateurs car leur entrée à la DN en 1993 est suivie d'une triple ou quadruple reconduction de leur mandat, ainsi que pour certaines, d'une participation à la Commission exécutive.

Ensuite, la victoire électorale municipale, à São Paulo, en 2000 et nationale en 2002 s'accompagne de la mise en place d'un féminisme d'État, concrétisant l'engagement du parti en matière de genre ainsi que l'influence de la communauté internationale sur la mise en ouvre de pratiques de «bonne gouvernance ». Ainsi la Coordenadoria da mulher, créée en 1988 sous l'administration municipale petista de Luiza Erundina mais mise en sommeil durant les mandats de Celso Pitta et Paulo Maluf, est réactivée. Un Secretaria especial de políticas para as mulheres ${ }^{35}$ ayant le statut de ministère, voit le jour en mars 2003. Cette inclusion des thématiques de genre au sein de l'arène institutionnelle classique convertit le SNF en réserve éventuelle

33 Dix-huit femmes ont été interrogées : il s'agissait d'élues ou de membres de l'administration petista ayant intégré le parti dès sa formation et participant aux divers secrétariats de femmes. Sur cet échantillon, $77 \%$ des femmes rencontrées avaient au minimum un diplôme de second cycle, $57 \%$ travaillaient dans l'enseignement et $25 \%$ dans les métiers du « social », c'est-à-dire de la santé ou de l'éducation.

34 Arlete Avelar Sampaio est députée estadual du district fédéral, Clara Ant est aujourd'hui une proche collaboratrice de Lula, Iriny Lopes est députée féderal.

35 Secrétariat spécial aux politiques pour les femmes. 
de cadres administratifs. Deux féministes historiques du parti, Tautau Godinho et Maria Luiza Da Costa, ont ainsi été appelées à présider la Coordenadoria da mulher à São Paulo.

De nombreuses défections de militantes féministes suivent la prise de pouvoir, municipale et nationale, du PT : contestation de la politique appliquée et/ou déception de ne pas avoir été rétribuée pour l'engagement au sein du parti? Plusieurs militantes petistas expliquent leur désengagement en mêlant ces deux variables:

«J'ai été secrétaire aux femmes au niveau municipal, national. J'ai été l'une des fondatrices du PT dans ma ville, dans le Minas Gerais [...] il y avait beaucoup de femmes dans les années 1980, 1990 au PT [. . .]. Mais progressivement, on s'est rendu compte que l'on était juste là pour balayer le sol [...] Donc on s'est mobilisé pour les quotas. Mais qu'est ce qui a changé? Le parti s'engage-t-il concrètement sur le thème des femmes? Et dès qu'il y a un enjeu de pouvoir, les femmes restent à la traîne ... donc à force d'être exclues, on va ailleurs [...] aujourd'hui, il y a plus d'espace pour les femmes dans les mouvements que dans le parti » (Membre de Fala Preta, mouvement de femmes noires).

Si l'on assiste, depuis quelques années, à la constitution d'une élite féminine, celleci est l'objet d'une forte remise en cause tant par l'oligarchie consolidée et majoritairement masculine, que par les femmes du parti.

En premier lieu, l'accession aux postes de direction, facilitée par l'adoption des quotas, soulève le problème de l'individuation: l'identité sexuée qui est renvoyée aux dirigeantes est souvent vécue de manière discriminante: est-ce «la place qui confère du pouvoir ou le pouvoir qui confère la place »? ${ }^{36}$ Les femmes élues sont sommées de donner la preuve de leur compétence afin de justifier le renouvellement des cadres dirigeants perçu comme artificiel par une grande partie des élites masculines. De plus, si cette mesure répond à une volonté d'élargir aux femmes l'accès aux instances de direction, elle n'a pas été accompagnée d'une redéfinition des conditions sociales de l'exercice du pouvoir. Par exemple, les revendications tenant au réaménagement de l'emploi du temps politique, destinées à prendre en compte les contraintes rencontrées par les militantes, n'ont pas été retenues. Les quotas ont donc permis aux femmes ayant déjà une certaine assise dans le parti de renforcer leur légitimité.

En second lieu, la contestation de l'«oligarchie » féminine naissante par les femmes du parti, repose sur deux types de critères: leur faible influence sur le programme petista; le manque de représentativité des multiples identités des femmes. Les femmes élues sont, tout d'abord, l'objet d'attentes de la part des féministes du parti, fondées sur un l'idéal de «représentation-figuration », selon lequel seuls les membres d'un groupe peuvent représenter les intérêts de ce dernier ${ }^{37}$. Dans cette perspective, une présence féminine devrait contribuer à accroître les politiques dirigées en faveur des femmes. Cependant, la naissance d'une élite fémi-

36 B. Marques-Pereira, «Enjeux et écueils de la représentation politique des femmes » in B. Marques-Pereira \& P. Nolasco (eds), La représentation politique des femmes en Amérique latine, Paris, l'Harmattan-GELA-IS, 2001 : 7-17.

37 Pour une analyse des arguments justifiant la représentation des «minorités », cf. A. Philips, The Politics of Presence, Oxford, Clarendon Press, 1995, 159 p.; I. Young, Inclusion and Democracy, Oxford, Oxford University Press, 2000, 304 p. et H.F. Pitkin, The Concept of Representation, Berkeley-Los Angeles, University of California, 1972, 2977nPoaded from Brill.com04/26/2023 12:32:47AM 
nine ne semble pas se traduire, nécessairement, par une inclusion des revendications de genre au sein du programme petista.

Puis, la représentativité des femmes politiques est fortement questionnée en raison des multiples identités des acteurs ${ }^{38}$. L'hétérogénéité des femmes a conduit la science politique à les considérer comme un groupe nominal, c'est-à-dire comme un ensemble d'individus partageant un caractère commun - le sexe. Néanmoins, l'identité de genre n'étant pas réductible aux identités sociales ou ethniques, pour ne citer qu'elles, les gender studies ont montré qu'elles méritaient une représentation politique propre. Il ne s'agit pas de remettre en question ce postulat, mais de l'approfondir. On observe, dans le cas du PT, que l'identité de genre des femmes politiques n'est pas suffisante pour fonder leur représentativité : celle-ci ne peut être pensée qu'en relation avec d'autres rapports de pouvoir. C'est parce que les élites féminines constituent un groupe relativement homogène, ne rendant pas compte des multiples identités des femmes, qu'elles sont contestées. Les principales critiques sont les suivantes :

- la sur-représentation des femmes blanches : les 15 et 16 avril 2004, j’ai assisté à la première rencontre nationale des femmes noires du PT. Celle-ci constitue l'amorce d'une autonomisation et d'une institutionnalisation de l'organisation des femmes noires au sein du parti. Ces dernières revendiquent tant un accès aux instances de directions partisanes et aux postes gouvernementaux, que l'engagement du parti sur un ensemble de thématiques;

- la sur-représentation des membres du courant majoritaire, l'Articulação (Articulation). Le secrétariat national connaît depuis quelques années une opposition croissante entre deux courants féministes: l'un défendant un «féminisme pratique », plus populaire et lié à la Marche mondiale des femmes, l'autre, un «féminisme stratégique ${ }^{39}$ », et proche du mouvement Articulação das mulheres brasileiras. L'homogénéité partisane jusque-là en vigueur au sein des secrétariats tend à s'estomper ;

- le désengagement des élues hétérosexuelles à l'égard des problématiques liées à l'homosexualité féminine.

L'analyse de R. Michels, certes quelque peu datée, met en lumière la nécessaire reconnaissance, par les militants des partis, de la légitimité des dirigeants, cette dernière étant une condition de leur maintien au pouvoir. Les élites féminines naissantes doivent, quant à elles, continuellement démontrer le bien-fondé de leur présence: elles sont d'autant plus contraintes de fournir la preuve de leur compétence, lorsqu'elles accèdent à un poste de direction, que leur élection se fait au détriment de dirigeants bien implantés, dont la légitimité n'est plus à prouver. Elles sont, en outre, l'objet d'attentes en matière de représentation des intérêts ; la réalisation partielle de ces dernières étant à l'origine de fortes critiques de la

${ }^{38}$ Sur la multiplicité des identités du sujet, $c f$. C. Mouffe, «Féminisme, citoyenneté et démocratie plurielle », in T.H. Ballmer-Cao et al. (eds), Genre et politique, Paris, Gallimard, 2001 : 176-197.

${ }^{39}$ Maxyne Molyneux distingue les revendications pratiques découlant des conditions concrètes d'attribution des rôles dans la division du travail en fonction du genre, et les revendications stratégiques découlant de l'analyse des rapports sociaux de sexe. Cité par S. Alvarez, Engendering democracy ..., op cit. : 54 . 
part des femmes du parti. Cette double remise en question symbolise les difficultés que les femmes rencontrent lorsqu'elles prétendent représenter l'universel.

Si les organisations politiques semblent ne pas pouvoir échapper à la loi d'airain de l'oligarchie, celle-ci doit être considérée de manière dynamique selon les arènes partisanes. Les groupes en interaction s'institutionnalisent et se professionnalisent de manière variable, ces processus n'étant néanmoins ni systématiques ni nécessaires.

L'organisation des femmes au sein du PT à São Paulo, en étant de plus en plus intégrée dans l'action institutionnelle du parti, suit l'évolution globale de ce dernier. On observe une certaine professionnalisation des militantes des secrétariats aux femmes ainsi que la naissance d'une élite féminine restreinte. Cependant, bien que ce phénomène symbolise une évolution des rapports de pouvoir entre les sexes au sein du parti, les femmes politiques restent soumises à une présomption d'illégitimité. Être femme représente bien une transgression en politique : les élues ne semblent pas pouvoir représenter l'universel, à la différence des élites masculines. Dès lors, si l'on peut parler de la création d'une élite féminine, celleci s'inscrit dans une reproduction des catégorisations binaires, caractérisant le masculin et le féminin, entre l'identique et le différent ${ }^{40}$ et dans le cas du PT du légitime et de l'illégitime.

Enfin, l'analyse de l'évolution des structures proprement féminine au sein du PT permet d'interroger les pratiques militantes de genre. Si le comportement des femmes petistas au sein du SNF corrobore d'abord l'existence de pratiques «genrées », et notamment moins compétitives, les distinctions tendent à s'atténuer lorsque ces mêmes espaces deviennent l'enjeu de position de pouvoir. Ce phénomène est-il seulement le fait de contraintes structurelles, les arènes partisanes et institutionnelles reposant sur un ensemble de normes reproduisant les discriminations de genre? Les pratiques distinctes des militantes du PT, particulièrement notables lors des premières années d'existence des secrétariats, doivent-elles être interprétées à l'aune d'une identité féminine particulière ou d'une position de dominées au sein de la structure partisane? N'existe-t-il pas également un certain «autoritarisme $»^{41}$ chez les femmes, permettant de comprendre l'évolution des secrétariats? Une telle recherche permettrait d'alimenter les discussions théoriques sur le mode féminin de «faire de la politique ».

Mai et septembre 2005

Marie-Hélène SÁ VILAS BOAS

Institut d'études politiques d'Aix-en-Provence Centre de Science politique comparative <msavilas@hotmail.com>

${ }^{40}$ Cf. F. Héritier, Masculin/Féminin II. Dissoudre la hiérarchie, Paris, Odile Jacob, 2002, 448 p.

${ }^{41}$ Le terme «autoritarisme» est ici pris dans le sens, très large, de reproduction des comportements de domination. Il fait référence au cycle de séminaire animé par le CSPG d'Aix-enProvence, sur l'autoritarisme. 